\title{
EXO modifies sucrose and trehalose responses and connects the extracellular carbon status to growth
}

\author{
Janina Lisso, Florian Schröder and Carsten Müssig* \\ Department Lothar Willmitzer, Max Planck Institute of Molecular Plant Physiology, Universität Potsdam, Potsdam, Germany
}

\section{Edited by:}

Bertrand Muller, Institut National de la Recherche Agronomique, France

\section{Reviewed by:}

Alessio Giovannelli, Italian National Research Council, Italy

Astrid Wingler, University College London, UK

\section{*Correspondence:}

Carsten Müssig, Department Lothar Willmitzer, Max Planck Institute of Molecular Plant Physiology,

Am Mühlenberg 1, 14476

Potsdam-Golm, Germany

e-mail:muessig@uni-potsdam.de
Plants have the capacity to adapt growth to changing environmental conditions. This implies the modulation of metabolism according to the availability of carbon (C). Particular interest in the response to the $\mathrm{C}$ availability is based on the increasing atmospheric levels of $\mathrm{CO}_{2}$. Several regulatory pathways that link the $\mathrm{C}$ status to growth have emerged. The extracellular EXO protein is essential for cell expansion and promotes shoot and root growth. Homologous proteins were identified in evolutionarily distant green plants. We show here that the EXO protein connects growth with $\mathrm{C}$ responses. The exo mutant displayed altered responses to exogenous sucrose supplemented to the growth medium. Impaired growth of the mutant in synthetic medium was associated with the accumulation of starch and anthocyanins, altered expression of sugar-responsive genes, and increased abscisic acid levels. Thus, EXO modulates several responses related to the $\mathrm{C}$ availability. Growth retardation on medium supplemented with 2-deoxy-glucose, mannose, and palatinose was similar to the wild type. Trehalose feeding stimulated root growth and shoot biomass production of exo plants whereas it inhibited growth of the wild type. The phenotypic features of the exo mutant suggest that apoplastic processes coordinate growth and $\mathrm{C}$ responses.

Keywords: EXO, growth, sugar response, trehalose, apoplast

\section{INTRODUCTION}

A major challenge for plants is the coordination of supply and demand of carbohydrates and other carbon (C) compounds during the diurnal cycle and under changing environmental conditions. Plants developed regulatory pathways that allow matching $\mathrm{C}$ consumption and availability. These pathways imply both longdistance and cell type-specific signaling mechanisms (Rolland et al., 2006). Rapid control of $C$ and energy homeostasis may primarily involve posttranslational modifications of proteins and allosteric control of enzymes, whereas transcriptional regulation and adjustment of growth mediate long-term adaptation.

As plants adapted to lower levels of ambient $\mathrm{CO}_{2}$ for millions of years, the current increase of atmospheric $\mathrm{CO}_{2}$ concentrations will affect crop yields via its effects on photosynthesis, plant metabolism, and water consumption (Leakey et al., 2009). Plant breeders possibly will be able to harness the $\mathrm{CO}_{2}$-enriched environment and increase yield of $\mathrm{C} 3$ crops, but there are many unanswered questions. Sink capacity (i.e., the transport, consumption, and storage of sugars and nutrients), maintenance of an optimal C/N balance, or photosynthetic capacity (e.g., Rubisco activity) may limit crop yield, but the regulatory processes at the basis of these physiological phenomena are poorly understood. Consequently, precise strategies to improve crops are difficult.

Although the rising atmospheric $\mathrm{CO}_{2}$ levels will impose farranging ecological and agricultural changes, a critical shortage of $\mathrm{C}$ and energy is a common short-term stress in plants. Low light, defoliation by mechanical injury or insect herbivory, or adverse environmental conditions such as flooding stress and hypoxia can result in poor $\mathrm{C}$ assimilation and ATP production.
Massive changes in enzymes activities, metabolic pathways, and gene expression patterns take place in response to $\mathrm{C}$ starvation and energy shortage (Gibon et al., 2009; Bailey-Serres et al., 2012). However, these adaptations primarily reflect stress responses, and the control of growth in dependency of $\mathrm{C}$ availability in the absence of stress may involve fine-tuning mechanisms that differ from short-term survival programs.

The analysis of the regulatory pathways that control C partitioning and starch turnover during diurnal cycles may provide more direct insights into the mechanisms linking $\mathrm{C}$ availability and growth (Stitt and Zeeman, 2012). For example, both too rapid and too slow mobilization of starch during the night can result in diminished growth rates. Rapid starch degradation causes $C$ starvation at the end of the night, and slow mobilization of starch during the night implies the retention of $\mathrm{C}$ reserves that are not used. The circadian clock is one factor that controls transitory starch accumulation, partly via the control of gene expression patterns (Graf et al., 2010; Farré and Weise, 2012; Ruts et al., 2012).

The evolutionary conserved protein kinase Snf1-Related Protein Kinase 1 (SnRK1) is a major regulator of energy homeostasis in plants. It controls numerous genes, metabolic pathways, and ultimately growth and development (Baena-González and Sheen, 2008). SnRK1 activity is controlled by the energy status, $\mathrm{C}$ availability, and additional physiological parameters. SnRK1 activity increases under low-energy conditions and promotes catabolic processes. One important regulative factor of SnRK1 activity is the signaling metabolite trehalose-6-phosphate (T6P) (Zhang et al., 2009). The T6P level is positively associated with 
the sucrose level (Lunn et al., 2006). High sucrose levels cause high T6P levels which inhibit SnRK1 activity. This inhibition may allow $\mathrm{C}$ consumption for anabolic processes and promote growth (Schluepmann et al., 2012). Conversely, low T6P levels under starvation conditions allow reprogramming of metabolism by high SnRK1 activity. T6P may also control starch synthesis via sucrose-dependent activation of AGPase (ADP-glucose pyrophosphorylase) (Lunn et al., 2006).

The target of rapamycin (TOR) pathway represents another highly conserved pathway that is critical for metabolic control and growth of cells. When nutrients are plentiful, TOR activates protein synthesis and growth. When nutrients are scarce, the TOR signaling pathway becomes inactive. This allows the induction of catabolic programs and, under severe starvation, autophagy of cell components. Presumably direct interactions between the plant TOR and SnRK1 signaling pathways exist, and the conservation of SnRKs and TOR in all eukaryotes suggests that these pathways represent an important regulatory module connecting nutrient sensing and cell growth (Smeekens et al., 2010; Robaglia et al., 2012).

The known signaling pathways described above are localized in the cytoplasm, are controlled by the nutrient and energy status of the cell, and control growth by modifying cell cycle progression, protein synthesis, and other processes. However, these pathways may need extensive input of various systemic and local signals to properly adjust metabolism to cellular needs and environmental conditions. For example, cell membrane sensors may sense physical cell wall properties, trigger enhanced delivery of cell wall components, and thus maintain cell wall integrity during growth and pathogen defense (Boisson-Dernier et al., 2011; Cheung and Wu, 2011; Levin, 2011; Wolf et al., 2012). Phytohormones interact with sugar responses, sink-source relations, and metabolism (Eveland and Jackson, 2012).

In addition to the regulatory pathways mentioned above, many mutants were identified that show altered responses to sugars (Rolland et al., 2006). Several of these mutants are also impaired in phytohormone biosynthesis or signaling. Examples include abscisic acid-deficient or abscisic acid-insensitive mutants (e.g., gin6/sun6/abi4, Arenas-Huertero et al., 2000; Huijser et al., 2000; aba2/sis4/gin1, Laby et al., 2000; Cheng et al., 2002), mutants with impaired ethylene production or impaired ethylene signaling (e.g., etr1-1, Zhou et al., 1998; sweetie, Veyres et al., 2008), mutants with altered brassinosteroid responses (e.g., bls1, Laxmi et al., 2004), and mutants with altered sensitivity to multiple phytohormones (e.g., prl1, Németh et al., 1998).

We identified the EXO and EXO-LIKE genes as brassinosteroid-responsive genes that control growth under different environmental conditions (Schröder et al., 2009). The EXL1 protein links $\mathrm{C}$ and energy metabolism to growth under suboptimal C availability (Schröder et al., 2011). Although EXL1 is required for adaptation to $\mathrm{C}$ - and energy-limiting growth conditions, it is largely irrelevant for growth and development under standard conditions (Schröder et al., 2011). EXL1 reduces BR-dependent growth under low $\mathrm{C}$ availability and presumably shuts down $\mathrm{C}$ and energy consumption in frame of a starvation response. The EXL2 and EXL4 proteins may have related functions (Schröder et al., 2012). EXO is required for growth under standard growth conditions (Schröder et al., 2009). Loss of EXO function resulted in reduced leaf size, fresh weight, dry weight, and root length. Epidermis, palisade, and spongy parenchyma cells of the exo mutant were smaller in comparison to the wild type.

The physiological and molecular mode of action of EXO and other members of the protein family is unknown. Here, we provide evidence that EXO modifies sugar response of seedling growth, sugar responsive gene expression, and sugar-dependent anthocyanin, starch, and ABA accumulation. The growth defects of the exo mutant are partly normalized by exogenous trehalose. We hypothesize that EXO links the extracellular $\mathrm{C}$ status with the intracellular $\mathrm{C}$ responses.

\section{MATERIALS AND METHODS PLANT MATERIALS AND GENERATION OF TRANSGENIC PLANTS}

The SALK_098602 line (Alonso et al., 2003) carried a T-DNA insertion in the EXO coding sequence and was named exo. The DNA insertion site, homozygosity of T-DNA insertion, and impaired gene expression were confirmed (Schröder et al., 2009). Accession Col-0 is the wild-type background and was used as control plants.

A 35S::EXO:HA overexpression construct was established using a Gateway-compatible vector. The EXO coding sequence was amplified using the primers forward $5^{\prime}$ CAC CCC TCT TTC ACT ATT ACA CTT TTC CT $3^{\prime}$ and reverse 5' GAC CAT AGT AGA GCA AGC CGA C 3'. Sequence analysis revealed $100 \%$ identity to the EXO cDNA sequence. The PCR fragment was cloned into the pENTR/D-TOPO (Invitrogen, Karlsruhe, Germany) vector, and was used to establish the 35S::EXO:HA fusion construct using the pGWB14 (Nakagawa et al., 2007) vector. The construct was transformed into exo mutant plants using the floral-dip method. Insertion of the construct and expression of EXO in exo/35S::EXO-HA plants were confirmed (Schröder et al., 2009).

\section{GROWTH CONDITIONS}

Seeds for growth experiments were derived from plants that were disseminated and grown in parallel in a greenhouse under standard conditions. Plants were grown on half-concentrated Murashige and Skoog (MS) medium supplemented with or without $0.5 \%$ sucrose and solidified with $0.4 \%(\mathrm{w} / \mathrm{v})$ agarose. After 2 days in the dark and at $4^{\circ} \mathrm{C}$, plants were transferred into a growth chamber with a long-day light regime (16 h day $\left[140 \mu \mathrm{mol} \mathrm{m} \mathrm{m}^{-2} \mathrm{~s}^{-1}, 22^{\circ} \mathrm{C}\right] / 8 \mathrm{~h}$ night $\left.\left[22^{\circ} \mathrm{C}\right]\right)$ and grown in a randomized manner. After 3 days, the germinated plants where transferred on new one-half concentrated Murashige and Skoog medium supplemented with different concentrations of sucrose, other sugars or sugar analogues and solidified with $0.4 \%(\mathrm{w} / \mathrm{v})$ agarose. The plants where then transferred back into the same growth chamber. Alternatively, plants were established in soil. Seeds were allowed to germinate and to grow for 2 weeks in controlled growth chambers ( 7 days: $16 \mathrm{~h}$ light $\left[140 \mu \mathrm{mol} \mathrm{m}^{-2} \mathrm{~s}^{-1}\right]$, $20^{\circ} \mathrm{C}, 75 \%$ relative humidity; $8 \mathrm{~h}$ night, $6^{\circ} \mathrm{C}, 75 \%$ relative humidity; thereafter 7 days: $8 \mathrm{~h}$ light $\left[140 \mu \mathrm{mol} \mathrm{m}^{-2} \mathrm{~s}^{-1}\right], 20^{\circ} \mathrm{C}, 60 \%$ relative humidity; $16 \mathrm{~h}$ night, $16^{\circ} \mathrm{C}, 75 \%$ relative humidity). Subsequently, plants were transferred to long-day conditions in 
a controlled growth chamber (16 h light [140 $\mu \mathrm{mol} \mathrm{m} \mathrm{m}^{-2} \mathrm{~s}^{-1}$ ], $20^{\circ} \mathrm{C}, 75 \%$ relative humidity; $8 \mathrm{~h}$ night, $16^{\circ} \mathrm{C}, 60 \%$ relative humidity). All genotypes were grown in the same chamber at the same time in a randomized manner.

\section{GENE EXPRESSION ANALYSIS}

After shoot mass quantification, the plant material was immediately frozen in liquid nitrogen and powderized. Analysis of transcript levels was conducted using the same shoot material that was used for other analyses (i.e., quantification of ABA, anthocyanin, and sugar levels, and determination of trehalase activity).

Total RNA was isolated using the Trizol reagent (Invitrogen). One microgram of total RNA was reverse transcribed with the RevertAid H Minus First Strand cDNA Synthesis Kit (Thermo Scientific) to generate first strand cDNA. Real-time RT-PCR was performed with the Maxima SYBR Green qPCR Master Mix (Thermo Scientific). The cycle threshold values of the eIF $1 \alpha$ control gene were subtracted from the respective cycle threshold values of the genes of interest. Other control genes gave similar results (Figures A4, A5). The expression value of the genes at $0.5 \%$ sucrose was arbitrarily set to 10 , and all other values were adjusted accordingly. Higher numbers indicate higher transcript levels. Primer sequences for quantitative RT-PCR are listed in the Table A1. All primer pairs amplified single products, as shown by the melting temperatures of the amplicons.

\section{MEASUREMENT OF ABA LEVELS}

$\mathrm{ABA}$ extraction was done at $4^{\circ} \mathrm{C}$ with dimmed light. $50 \mathrm{mg}$ powdered shoot tissue were subjected to lyophilisation for $24 \mathrm{~h}$. Tissue was suspended in $0.5 \mathrm{ml}$ extraction buffer $(\mathrm{MeOH}$ containing $2.5 \mathrm{mM}$ citric acid monohydrate and $0.5 \mathrm{mM}$ butylated hydroxytoluene). The extract was incubated for at least $20 \mathrm{~h}$ in the dark at $4^{\circ} \mathrm{C}$ under shaking conditions and centrifuged at $1500 \times \mathrm{g}$ for $15 \mathrm{~min}$ at $4^{\circ} \mathrm{C}$. The supernatants were recovered and $2 \mathrm{ml}$ $62.5 \%$ extraction buffer $/ 28.5 \% \mathrm{MeOH}$ was added. C18 Sep-Pak cartridges (Waters, Eschborn, Germany) were equilibrated with $2 \mathrm{ml}$ extraction buffer and subsequently with $1 \mathrm{ml} 70 \%$ extraction buffer $/ 30 \% \mathrm{MeOH}$. Supernatants were passed through a C18 Sep-Pak cartridge. $1 \mathrm{ml} 70 \% \mathrm{MeOH}$ was loaded onto the cartridge and flow-throughs were united. Additional elutions were analyzed exemplarily to check for residual ABA on the cartridge. The eluates were dried in a lyophilizer. Dried samples were resuspended in $1 \mathrm{ml} 50 \mathrm{mM}$ Tris buffered saline $\mathrm{pH} 7.5$ (TBS)/MeOH (10:1). Dilutions in TBS of each sample extract or ABA standard (Duchefa) were subjected to analysis by an enzyme immunoassay using the PGR1 kit (Sigma-Aldrich) according to the manufacturer's instruction. The ABA standard was assumed to be a mixture of equal amounts of the (S)-2-cis and (S)-2-trans form of ABA.

\section{ANALYSIS OF ANTHOCYANIN LEVELS}

Fifty milligram of shoot material were extracted for $1 \mathrm{~d}$ at $4^{\circ} \mathrm{C}$ in $1 \mathrm{ml}$ of $1 \%(\mathrm{v} / \mathrm{v})$ hydrochloric acid in methanol. The mixture was centrifuged at $18000 \times \mathrm{g}$ for $15 \mathrm{~min}$ and the absorbance of the supernatant was measured at 530 and $657 \mathrm{~nm}$. Relative anthocyanin concentrations were calculated with the formula
[A530 - $(0.25 \times$ A657) $]$ to correct for chlorophyll and its degradation products present in the extract.

\section{DETERMINATION OF SUGARS}

Plants were harvested at the middle of the light period. Quantification of sugars was conducted using the same shoot material that has been applied to gene expression analysis, quantification of ABA, anthocyanin, and trehalase activity. $50 \mathrm{mg}$ of shoot material were extracted twice with $80 \%$ ethanol at $95^{\circ} \mathrm{C}$ for $30 \mathrm{~min}$, followed by one extraction with $50 \%$ ethanol at $95^{\circ} \mathrm{C}$ for $30 \mathrm{~min}$. The supernatant was used for the determination of glucose, fructose and sucrose by measuring the difference of absorbance at $340 \mathrm{~nm}$ in buffer $(75 \mathrm{mM} \mathrm{HEPES} / \mathrm{KOH}$ $\mathrm{pH} 7,2.3 \mathrm{mM} \mathrm{MgCl}, 2.3 \mathrm{mM}$ ATP, $1 \mathrm{mM}$ NADP and glucose6-phosphate dehydrogenase) after sequentially adding of hexokinase, phosphoglucose isomerase, and invertase. For starch determination, the pellets of the ethanol extraction were solubilized by heating them to $95^{\circ} \mathrm{C}$ in $0.1 \mathrm{M} \mathrm{NaOH}$ for $30 \mathrm{~min}$. After acidification with an $\mathrm{HCl} /$ sodium-acetate mixture $\mathrm{pH} 4.9$, part of the suspension was digested overnight with amyloglucosidase and $\alpha$-amylase. The glucose content of the supernatant was then used to assess the starch content of the sample by measuring the difference in absorbance at $340 \mathrm{~nm}$ after adding hexokinase in the same buffer mentioned above.

\section{TREHALASE ACTIVITY}

Aliquots of $70 \mathrm{mg}$ frozen shoot material were resuspended in $1 \mathrm{ml}$ cold extraction buffer (0.1 M MES-KOH pH 6, 1 mM EDTA, $1 \mathrm{mM}$ phenylmethylsulfonyl fluoride, $1 \% \mathrm{w} / \mathrm{v}$ polyvinylphenol, $0.01 \%$ Triton X-100, and $1 \mathrm{mM} \mathrm{DTT}$ ) at $4^{\circ} \mathrm{C}$. The suspension was cleared by centrifugation at $13000 \times \mathrm{g}$ and $4^{\circ} \mathrm{C}$ for $10 \mathrm{~min}$. Removal of sugars and the subsequent trehalase assay were conducted as described in Delatte et al. (2011).

\section{RESULTS}

\section{EXO ALTERS RESPONSES TO EXOGENOUS SUGARS}

To test growth in the presence of exogenous sugar, wild-type and exo plants were grown in half-concentrated Murashige and Skoog (MS) medium supplemented with different sucrose concentrations. When cultivated on $0.5 \% \mathrm{w} / \mathrm{v}$ sucrose, exo produced $47 \%$ of the wild-type biomass (Figure 1A). This approximates the growth defect of soil-grown exo plants (Figure A1 and Schröder et al., 2009). A lower sugar concentration such as $0.2 \% \mathrm{w} / \mathrm{v}$ sucrose caused reduced growth of the wild type, but barely affected growth of exo plants (Figure 1A). A high sugar concentration (3\% sucrose) caused a 19 and 53\% reduction in shoot biomass growth of wild-type and exo plants, respectively, indicating that exo is more sensitive to high sugar levels in comparison to the wild type. Overexpression of an HA-tagged EXO protein under control of the $35 \mathrm{~S}$ promoter in the exo mutant largely normalized the sucrose response (Figure 1A).

Addition of sucrose increases the osmolarity of the growth medium and conceivably causes osmotic stress. In order to test osmotic stress sensitivity, plants were grown on medium supplemented with $0.5 \%$ sucrose and mannitol. Mannitol is a sugar alcohol that is widely used as osmotic agent in plant research. The exo mutant produced about $45 \%$ of the wild-type biomass 

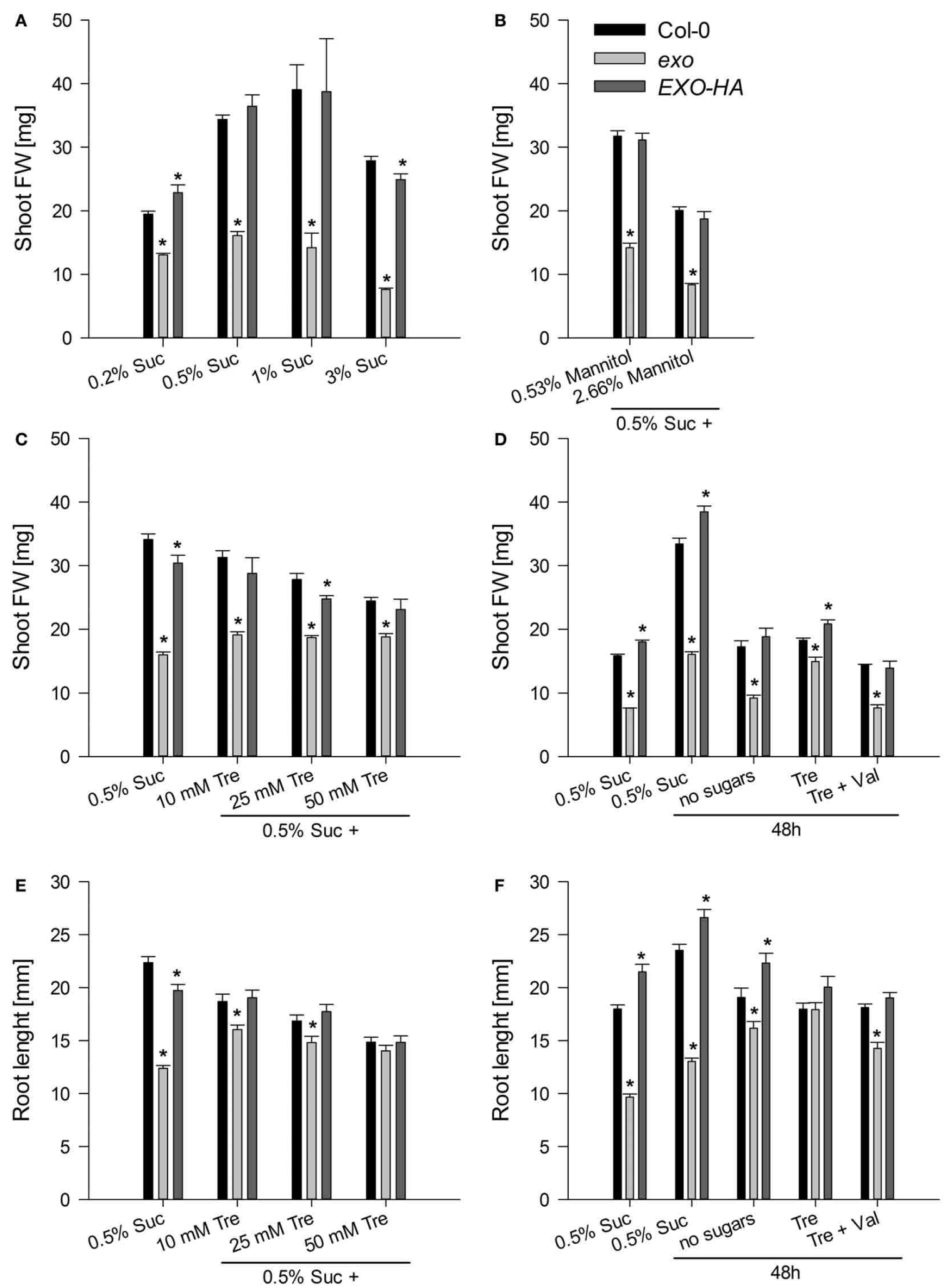

FIGURE 1 | Fresh weight of shoots and length of roots. (A) Wild-type, exo, and exo/35S::EXO-HA plants (termed EXO-HA) were grown on half-concentrated MS medium in the presence of different sucrose concentrations for 14 days. Eight plants were combined for each weight

determination. Data are means of 5 values \pm SE. Mutant values denoted with an asterisk are significantly different from those of their wild type ( $t$-test, $P<0.001$ ). Data are representative of three independent experiments with (Continued) 


\section{FIGURE 1 | Continued}

similar results. (B) Plants were established in half-concentrated MS medium for 5 days and transferred to medium supplemented with $0.5 \% \mathrm{w} / \mathrm{v}$ sucrose plus 0.53 or $2.66 \% \mathrm{w} / \mathrm{v}$ mannitol to gain osmolarities of the medium that approximate 1 and $3 \% \mathrm{w} / \mathrm{v}$ sucrose, respectively. Data are representative of three independent experiments with similar results. (C) Plants were established as described in (B) and transferred to half-concentrated MS medium supplemented with $0.5 \%$ sucrose plus $0,10,25$, or $50 \mathrm{mM}$ trehalose for 9 days. Data are representative of three independent experiments with similar results. (D) Plants were established in half-concentrated MS medium supplemented with $0.5 \%$ sucrose. 12 -day-old plants were transferred to medium supplemented with $0.5 \%$ sucrose, no sugar, $25 \mathrm{mM}$ trehalose (Tre), or $25 \mathrm{mM}$ Tre and $10 \mu \mathrm{M}$ validamycin $\mathrm{A}(\mathrm{Val})$, a potent trehalase inhibitor, and harvested 2 days later. Data are representative of three independent experiments with similar results. (E) Root length of the same plants as described in (C). (F) Root length of the same plants as described in (D). in the presence of $0,0.53$, and $2.66 \% \mathrm{w} / \mathrm{v}$ mannitol. That resembles growth inhibition of exo on medium with $0.5 \%$ sucrose only. Mannitol similarly impaired growth of wild-type and exo plants. Thus, the mutant was not hypersensitive to the increased osmotic concentration in the growth medium.

\section{SUGAR-RESPONSIVE GENES REFLECT SUCROSE-HYPERSENSITIVITY OF EXO}

Sugar supply affects the expression of a large set of genes. Expression levels of 24 genes were analyzed in response to exogenous sucrose by means of real-time RT-PCR.

Six starch-related genes were tested, namely APL3 and APL4 (encoding regulatory subunits of ADP-glucose pyrophosphorylase), BAM3 (also termed $B M Y 8$ or $c t B M Y$, encoding a plastidial $\beta$-amylase), BAM5 (also termed $B M Y 1$ or $R A M 1$, encoding a cytosolic $\beta$-amylase), SEX1 (also termed GWD1, encoding a plastidial $\alpha$-glucan water dikinase involved in starch degradation), and GLT1 (encoding a plastidial glucose translocator) (Sebastian and Zeeman, 2012). APL3, APL4, and BAM5 expression was induced by exogenous sugar in wild-type and exo plants, but expression levels in exo were consistently lower. BAM3, SEX1, and GLT1 transcript levels were not induced in response to sucrose, but also were lower in exo plants (Figure 2). Thus, genes involved in the synthesis and degradation of starch were weaker expressed in exo. Anyhow, reduced expression of genes involved in starch biosynthesis such as APL3 and APL4 presumably did not limit starch biosynthesis, because starch levels were significantly higher in exo in the presence of different sucrose (Figure 4A) and trehalose concentrations (Figures 4B,C). Glucose, fructose, and endogenous sucrose levels were not significantly different from the wild type (data not shown).

The BFRUCT1, CAB1, DIN1, DIN6, DIN10, RBCS1A, and SUC2 genes have diverging and partly unknown functions in plants, but a common feature is their control by sugars. $C A B 1$ (also termed LHCB1.3, encoding a subunit of light-harvesting complex II), DIN1 (also termed SEN1, senescence associated gene with unknown function), DIN6 (also termed ASN1, encoding an asparagine synthase), DIN10 (glycosyl hydrolase), and RBCS1A (Rubisco small subunit) expression was repressed by sucrose in both genotypes, but transcript levels were consistently lower in exo (Figure 3). Expression of the BFRUCT1 gene (also termed $C W I N V 1$, encoding a cell wall invertase) was induced by sucrose. In contrast to the other tested genes, BFRUCT1 transcript levels were slightly elevated in exo at 0.2, 0.5, and 1\% sucrose (Figure 3 ). The SUC2 gene encodes a sucrose transporter that is essential for phloem loading. SUC2 transcript levels were slightly lower in exo in the presence of sucrose. Omission of sucrose normalized SUC2 expression in the mutant (Figure 3).
Introduction of the 35S::EXO-HA construct into exo plants restored wild-type expression levels of both the starch-related and the sugar-responsive genes (Figures 2, 3).

The KIN10 (SnRK1.1) and KIN11 (SnRK1.2) kinases and the bZIP11 and ABI4 transcription factors play pivotal roles in the control of metabolism. Transcript levels of these genes were tested in wild-type and exo plants. Only minor or no differences were observed (Figure A2). We also tested the transcript levels of genes involved in trehalose metabolism. Apart from TPS1, transcript levels were similar in wild-type and exo plants (Figure A3).

The EXO gene itself is controlled by the $\mathrm{C}$ status. We previously reported that EXO expression is induced in the night, by $\mathrm{C}$ starvation, and by a low atmospheric $\mathrm{CO}_{2}$ concentration (Schröder et al., 2011). In line with these findings, EXO transcript levels were repressed by exogenous sucrose (Figure 5A). Trehalose feeding caused stronger EXO expression (Figures 5B,C).

\section{ABA AND ANTHOCYANIN LEVELS ARE ELEVATED IN EXO}

Sugars interact with phytohormones to control adaptation to stress and growth. A relationship between ABA and sugar responses was identified by means of mutant analyses. For example, sugar supply causes ABA accumulation in Arabidopsis seedlings (Arenas-Huertero et al., 2000; Huijser et al., 2000; Laby et al., 2000; Cheng et al., 2002). ABA levels were measured in exo and wild-type plants grown in medium supplemented with 0.2 , 0.5 , and $1 \%$ sucrose. ABA levels in exo were approximately $90 \%$ above the wild-type level (Figure 6A). In order to test whether ABA sensitivity was also altered in exo, the effects of synthetic ABA on root length and shoot biomass were analyzed. Four-day old seedlings were transferred to medium supplemented with different ABA concentrations as described in Lisso et al. (2011). Shoot biomass of exo seedlings in response to exogenous ABA was similarly impaired in comparison to the wild type (data not shown). In contrast to wild-type roots, exo roots were barely affected by exogenous ABA (Figure 6B).

Sucrose is an effective inducer of anthocyanin production in Arabidopsis seedlings through the activation of biosynthetic genes (Teng et al., 2005; Das et al., 2012).

Anthocyanin levels were elevated in the exo mutant at all tested sucrose levels (Figure 7A). On $0.5 \%$ sucrose, exo exhibited approximately a twofold anthocyanin concentration in comparison to the wild type. The complemented exo mutant had approximately wild-type anthocyanin levels.

\section{SUGAR ANALOGUES TRIGGER WILD-TYPE GROWTH RESPONSES IN EXO}

The effects of 2-deoxy-glucose, mannose, and palatinose on biomass production were tested. 2-deoxy-glucose is a glucose 


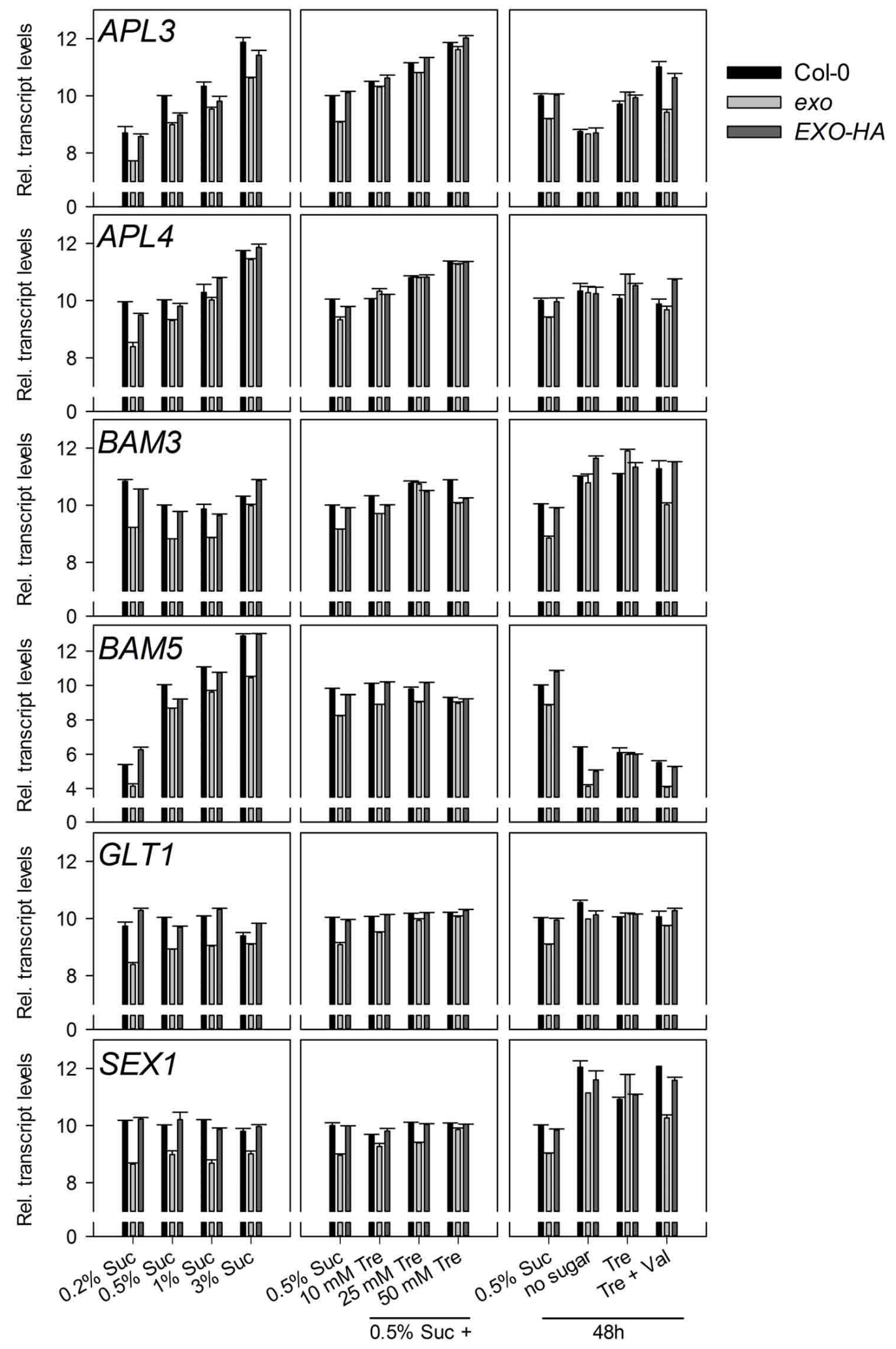

FIGURE 2 | Expression of starch-related genes. Quantitative RT-PCR analysis of $A P L 3, A P L 4, B A M 3, B A M 5, G L T 1$, and $S E X 1$ transcript levels. Wild-type, exo, and exo/35S::EXO-HA plants were grown on

half-concentrated MS medium supplemented with different sucrose concentrations for 14 days. Alternatively, plants were established in medium supplemented with $0.5 \%$ sucrose for 5 days and transferred to medium with $0.5 \%$ sucrose and $0,10,25$, or $50 \mathrm{mM}$ trehalose. For short-term treatments, (Continued) 


\section{FIGURE 2 | Continued}

12-day-old seedlings were transferred to plates supplemented with $0.5 \%$ sucrose, no sugar, $25 \mathrm{mM}$ trehalose (Tre), or $25 \mathrm{mM}$ trehalose plus $10 \mu \mathrm{mol}$ validamycin A (Tre + Val) and shoot material was harvested 2 days later at the middle of the light period. The cycle threshold values of the elF1 $\alpha$ control gene were subtracted from the respective cycle threshold values of the genes of interest. The expression value of the genes at $0.5 \%$ sucrose was arbitrarily set to 10 , and all other values were adjusted accordingly. Higher numbers indicate higher transcript levels. A difference of one unit indicates a 2-fold change. Error bars indicate SE of the gene of interest in three technical replicates. Data are representative of three independent experiments with similar results. analogue that becomes phosphorylated by hexokinases and blocks seedling development (Gibson, 2000). Mannose is an intermediate in sugar metabolism, and also acts as a glucose analogue that is phosphorylated by hexokinases. Feeding mannose inhibits development of Arabidopsis seedlings (Pego et al., 1999). Palatinose is a non-metabolisable structural sucrose isomer (Loreti et al., 2001). The observed relative growth responses to the three compounds were identical in exo and the wild type in comparison to the control situation, $0.5 \%$ sucrose. No differences in the relative growth inhibition were observed (Figure 8), suggesting that major hexose- and sucrose-dependent signaling pathways are intact in exo.

\section{EXOGENOUS TREHALOSE LARGELY RESCUES THE EXO MUTANT}

Trehalose is a glucose disaccharide that has physiological effects on growth and carbon allocation in plant seedlings. In addition, trehalose was reported to enhance abiotic stress resistance in plants. However, trehalose presumably does not function as compatible solute and stress protectant in Arabidopsis, because the endogenous concentrations are low (Vogel et al., 2001). Trehalose feeding inhibits growth of Arabidopsis seedlings, causes anthocyanin accumulation, and alters starch levels in several plant organs (Wingler et al., 2000; Aghdasi et al., 2010). The mode of action of growth inhibition includes the accumulation of the signaling compound T6P (Schluepmann et al., 2004).

Plants were established on medium supplemented with $0.5 \%$ sucrose. After 5 days, plants were transferred on medium supplemented with $0.5 \%$ sucrose and 0 to $50 \mathrm{mM}$ trehalose. In the absence of trehalose, exo plants produced $47 \%$ of the biomass of the wild type (Figures 1A,C). Addition of trehalose impaired growth of the wild type. For example, addition of $25 \mathrm{mM}$ trehalose in the presence of $0.5 \%$ sucrose caused about $20 \%$ reduction in biomass production and root length (Figures 1C,E). In contrast, addition of trehalose had positive effects on exo plants and partly normalized shoot and root growth (Figures 1C,E).

The inhibitory effect of trehalose is curbed by sugars, because growth of wild-type seedlings on trehalose is restored if sucrose is supplied simultaneously with trehalose (Schluepmann et al., 2004). To investigate the effect of trehalose on growth in the absence of sucrose, and to assess the full impact of trehalose, established seedlings were transferred to different media and allowed to grow for 2 days. In the presence of $0.5 \%$ sucrose, all genotypes showed shoot and root growth during the two-day period, demonstrating that the transfer process to new growth medium is not destructive (Figures 1D,F, see outermost left columns). The absence of sucrose resulted in $48 \%$ and $42 \%$ shoot biomass reduction in wild-type and exo plants, respectively (Figure 1D). Root growth of wild-type plants also was reduced in the absence of sucrose. In contrast, exo root length was elevated in the absence of sugars (Figure 1F). Supply of trehalose in the absence of sucrose largely normalized exo shoot and root growth, but did not positively affect wild-type growth (Figures 1D,F).

The rescue of the growth phenotype by exogenous trehalose was also reflected at the molecular level. Most starch-related and sugar-responsive genes were normally expressed in exo upon trehalose feeding (Figures 2, 3). Short-term treatments with $25 \mathrm{mM}$ trehalose in the absence of sucrose normalized anthocyanin levels in the mutant (Figure 7C). The altered response of exo to trehalose becomes also evident by comparing the starch levels. The wild type accumulated starch in response to trehalose, whereas exo starch levels did not increase upon long-term trehalose (Figure 4B) and partly normalized to the wild-type level upon short-term trehalose application (Figure 4C). Thus, growth of the exo mutant is promoted rather than impaired by trehalose.

\section{VALIDAMYCIN A PROHIBITS RESCUE OF EXO BY TREHALOSE}

Arabidopsis trehalase occurs in multiple tissues and is highly active in flowers and siliques. Low trehalase activity was detected in roots (Müller et al., 2001). The Arabidopsis genome encodes a single trehalase termed TRE1 that is a plasma membrane-bound enzyme with extracellular activity (Frison et al., 2007). Altering trehalase activity barely changed T6P levels in Arabidopsis, indicating that the altered trehalose content caused the phenotypic differences (Van Houtte et al., 2013).

Trehalase activity in exo was slightly reduced in medium supplemented with $0.5 \%$ sucrose (Figure 9A). Long-term trehalose supply in the presence of $0.5 \%$ sucrose induced trehalose activity in wild-type and exo plants. Higher trehalose levels completely normalized trehalase activity levels in exo (Figure 9A). Short-term trehalose supply in the absence of sucrose caused a massive induction of trehalase activity, most pronouncedly in exo (Figure 9A). Thus, trehalose feeding was associated with the induction of trehalase activity.

Validamycin A ( Val) is a strong inhibitor of trehalases in plant tissues (Müller et al., 2001). The simultaneous supply of Val prohibited trehalose-dependent normalization of exo shoot growth (Figure 1D), root growth (Figure 1F), and transcript levels (Figures 2, 3). Since the inhibition of trehalase prevents cleavage of trehalose into glucose, the extracellular generation of glucose apparently is essential for the normalization of growth and gene expression patterns in exo.

\section{DISCUSSION \\ THE APOPLASTIC EXO PROTEIN MODIFIES INTRACELLULAR SUGAR RESPONSES}

The exo loss-of-function mutant is characterized by reduced leaf and root growth. EXO is essential for cell expansion in leaves 


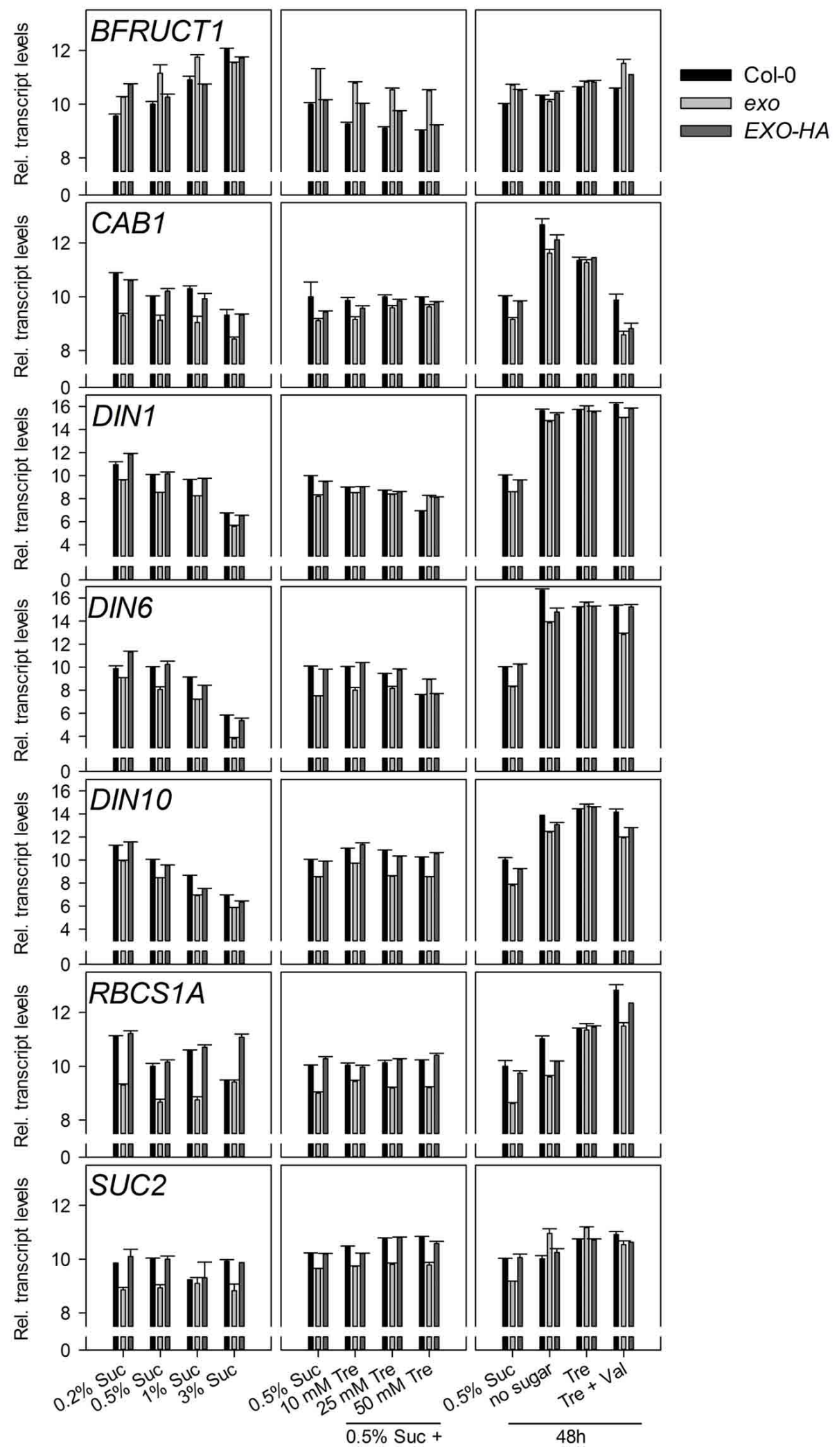

FIGURE 3 | Expression of sugar-responsive genes. Quantitative RT-PCR analysis of BFRUCT1, CAB1, DIN1, DIN6, DIN10 RBCS1A, and SUC2 transcript levels in wild-type, exo, and exo/35S::EXO-HA plants shoots. Technical details are as given for Figure 2. Data are representative of three independent experiments with similar results. 

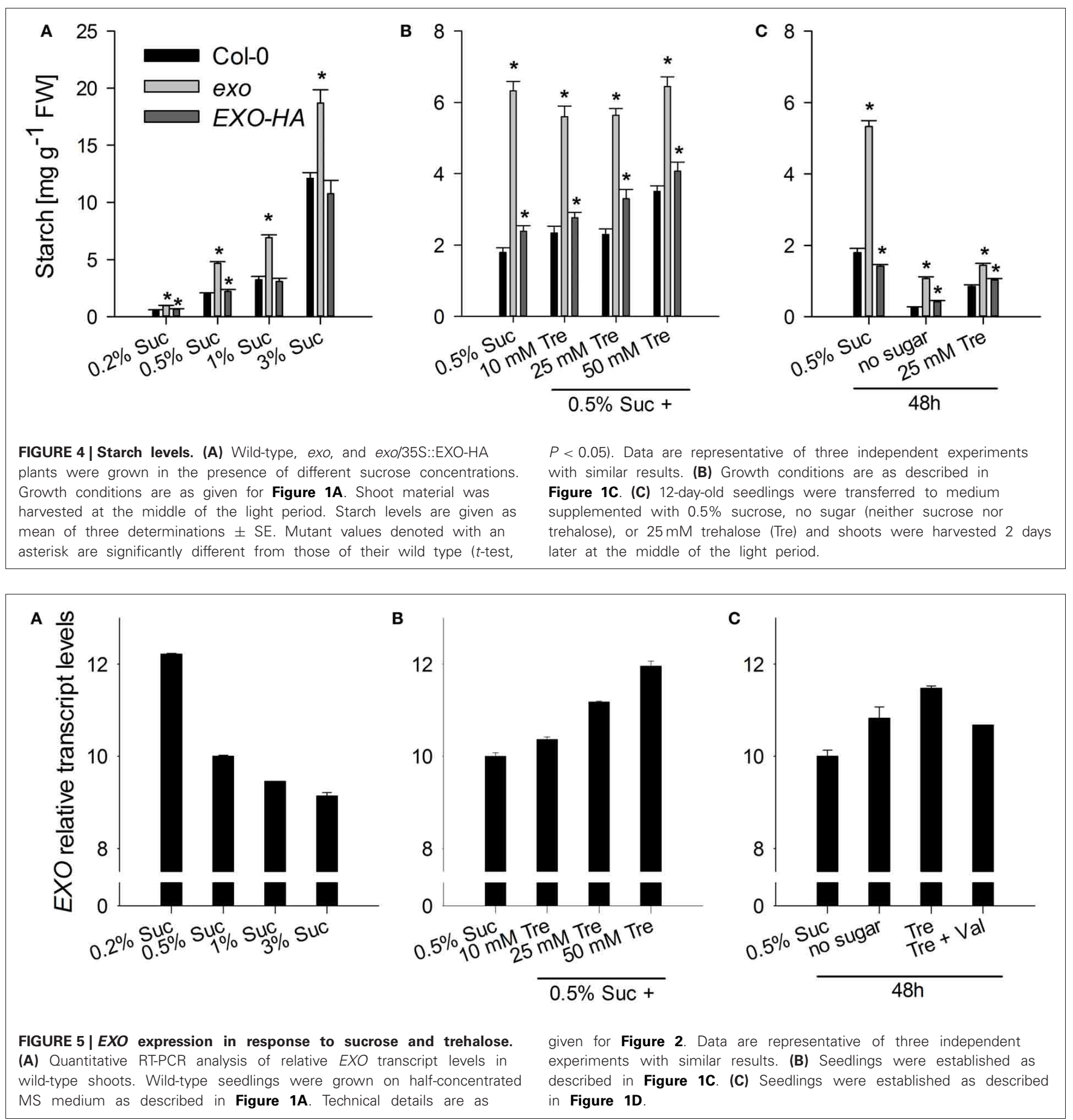

because epidermis, palisade and spongy parenchyma cells were smaller in exo in comparison to the wild type (Schröder et al., 2009). The highly similar EXO-LIKE1 (EXL1) protein promotes growth under C-limiting conditions (Schröder et al., 2011, 2012). The characteristic feature of the EXO and EXO-LIKE proteins is the PHI1 conserved region (Pfam entry PF04674). Its putative Nterminal targeting sequence directs the protein into the apoplast (Schröder et al., 2009). The remaining primary structure does not show similarity to other known protein domains.
The findings shown here suggest that EXO integrates the apoplastic $C$ status such as the level of sugars or sugarderived signals, intracellular sugar responses, and growth. Loss of EXO function caused altered sucrose response of seedling growth (Figure 1), suggesting that the exo mutant is impaired in the transport, perception, or signaling of sugars. ABA and anthocyanin levels are associated with the C status (Arenas-Huertero et al., 2000; Cheng et al., 2002; Teng et al., 2005; Das et al., 2012). The growth 


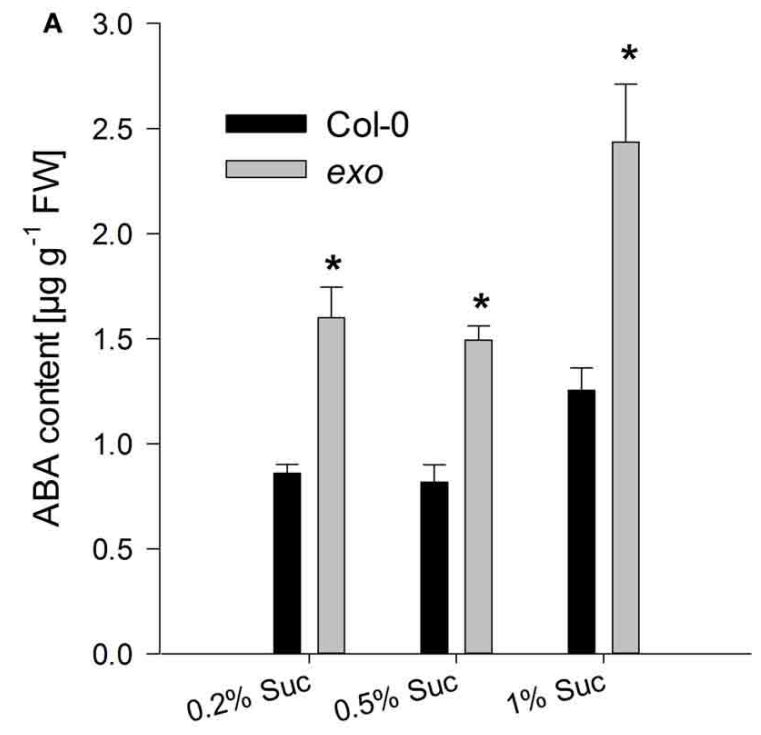

FIGURE 6 | ABA levels and root growth in response to exogenous ABA. (A) Wild-type and exo plants were grown on half-concentrated MS medium in the presence of different sucrose concentrations for 14 days. Shoot material was analyzed. Results are mean of three determinations \pm SE. Mutant values denoted with an asterisk are significantly different from those of their wild type ( $t$-test, $P<0.001$ ). Data are representative of three independent experiments with similar results. (B) Wild-type, exo, and

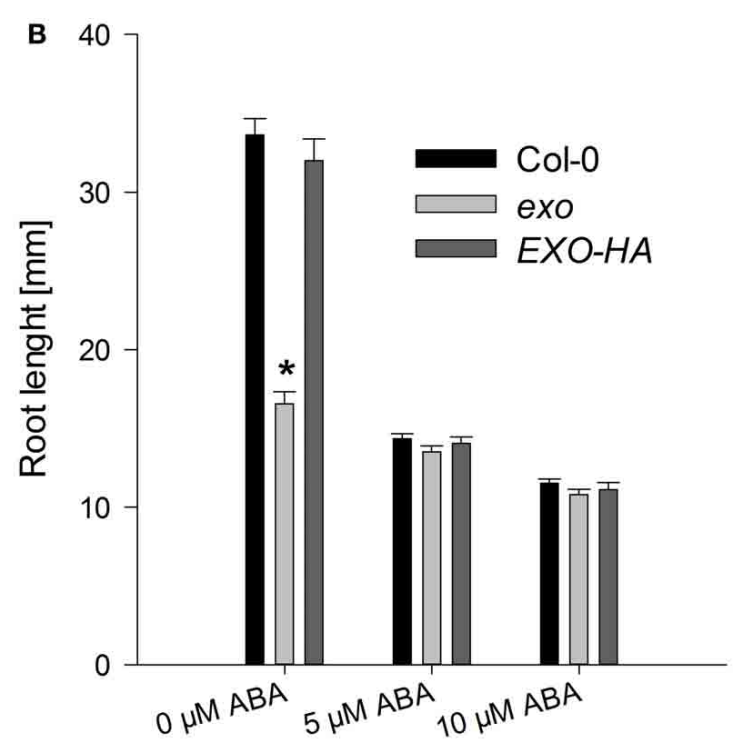

exo/35S::EXO-HA seeds were allowed to germinate in medium supplemented with $0.5 \%$ sucrose. 5 -day-old seedlings were transferred to the same medium supplemented with 0,5 , or $10 \mu \mathrm{M}$ ABA. Root length of 14-day-old plants was determined and is given as mean of 40 roots \pm SE. Mutant values denoted with an asterisk are significantly different from those of their wild type ( $t$-test, $P<0.001$ ). Data are representative of three independent experiments with similar results.

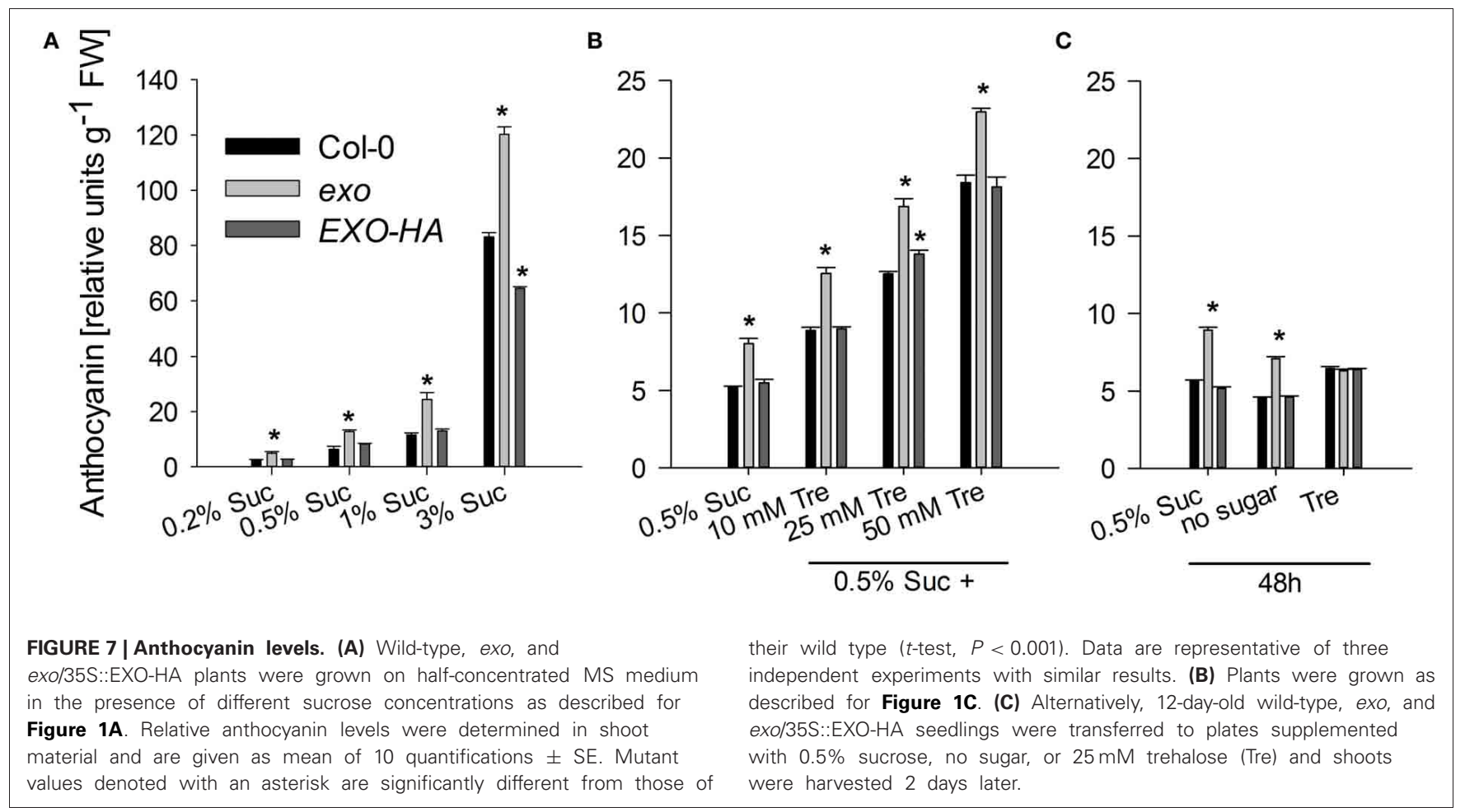

defect of exo is accompanied by elevated ABA (Figure 6), anthocyanin (Figure 7), and starch concentrations (Figure 4). Expression of several sugar-related genes was weaker in exo (Figures 2, 3).

\section{EXOGENOUS TREHALOSE COMPLEMENTS THE EXO MUTANT}

The localization of EXO in the apoplast and wild-type responses to 2-deoxy-glucose and mannose exclude a role as component in hexokinase-dependent signaling pathways. 


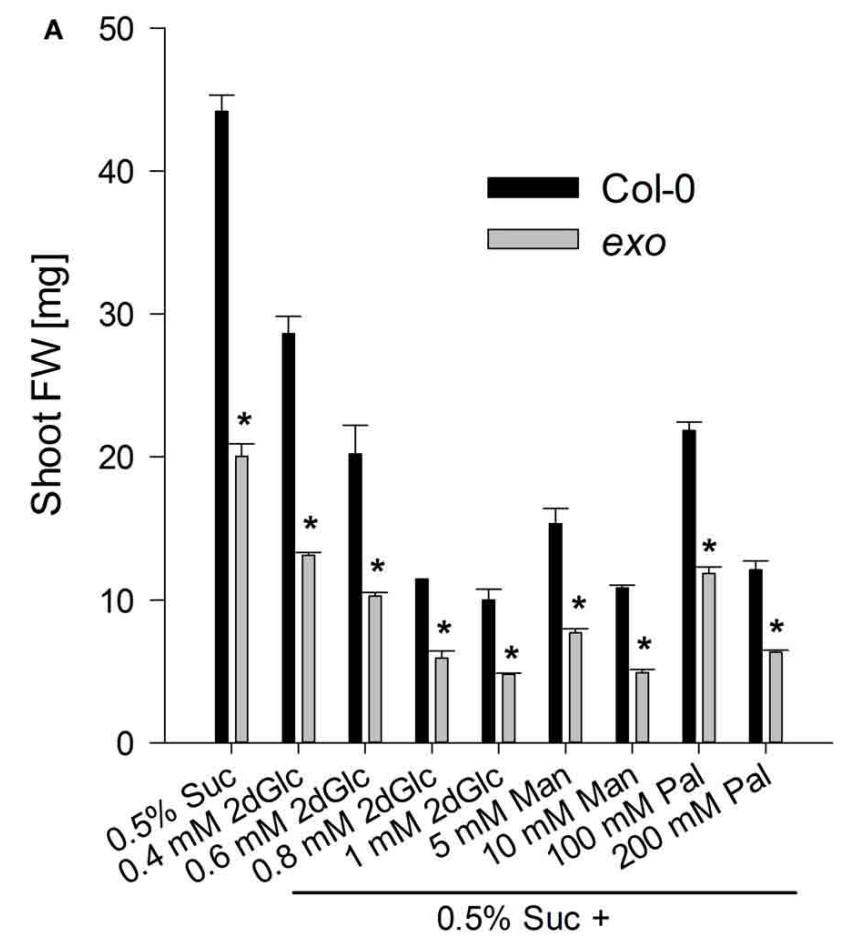

FIGURE 8|Shoot biomass in the presence of sugar analogues. Wild-type and exo plants were established in half-concentrated MS medium for 5 days and transferred to medium supplemented with $0.5 \%$ sucrose and different concentrations of 2-deoxy-glucose (2dGlc), mannose (Man), or palatinose (Pal). (A) Fresh weight of shoots. Seven plants were weighed together and results are mean of five

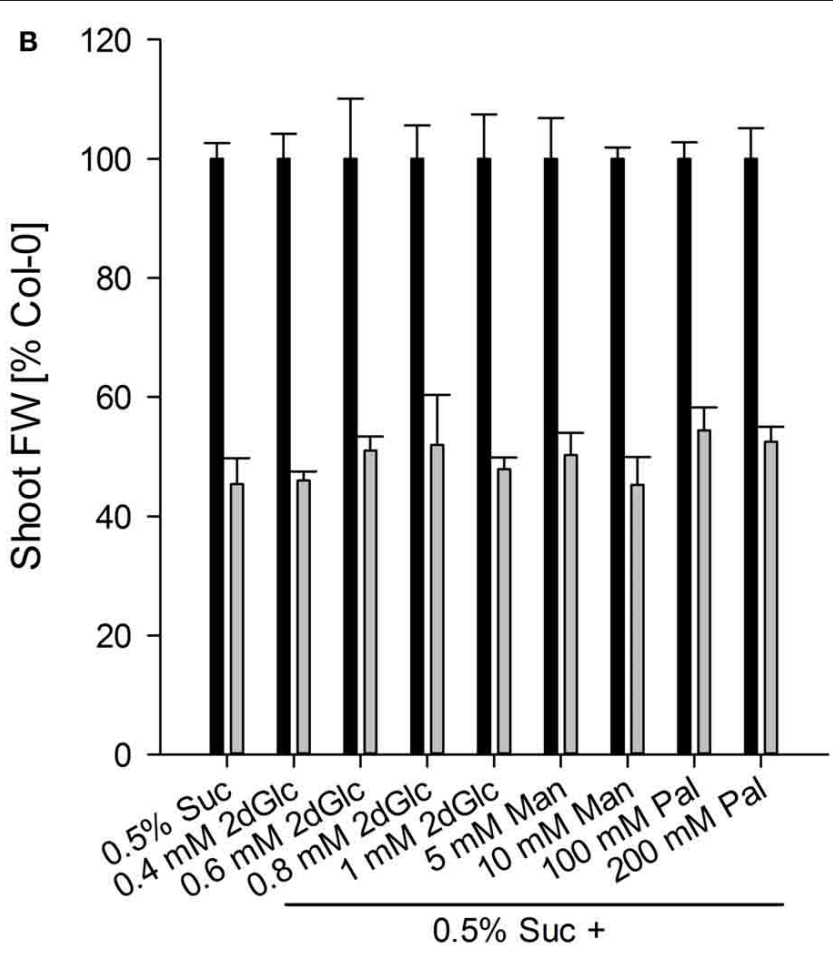

single values \pm SE. Mutant values denoted with an asterisk are significantly different from those of their wild type ( $t$-test, $P<0.001$ ). Data are representative of three independent experiments with similar results. (B) Relative fresh weight calculated from data in Figure 8A. Percentage changes were not significantly different in exo in comparison to the wild type.
An interaction with plasmamembrane glucose transporterlike sensors or G-protein coupled receptors that represent well-characterized signaling pathways in yeast is speculative, because these systems have been poorly described in plants (Rolland et al., 2006).

Interestingly, the phenotypic changes of exo were largely normalized by exogenous trehalose. The disaccharide slightly stimulated growth (Figures 1C-F), normalized sugar-related gene expression patterns (Figures 2, 3), and reduced anthocyanin levels (Figure 7). Thus, either trehalose itself or trehalose-derived compounds complement the exo mutant. Two lines of arguments suggest that extracellular trehalose-hydrolysis is essential for the complementation. First, complementation of the mutant by trehalose feeding was associated with enhanced trehalase activity (Figure 9). Second, simultaneous feeding of the trehalase inhibitor validamycin A reversed the positive trehalose effects (Figures 1-3).

Exogenous trehalose usually inhibits Arabidopsis seedling growth. Intracellular T6P accumulation was identified as a major cause for this (Schluepmann et al., 2004). Plants with enhanced trehalase activity grow better on trehalose. This was demonstrated for plants expressing an E. coli trehalase in the cytoplasm (35S::treF) and for plants expressing the Arabidopsis TRE1 enzyme (35S::TRE1) (Schluepmann et al., 2004; Aghdasi et al., 2010; Delatte et al., 2011; Van Houtte et al., 2013). 35S::treF plants hydrolyze trehalose that has entered the cell, whereas 35S::TRE1 seedlings could metabolize trehalose before it passes the plasma membrane. Although 35S::treF plants displayed higher trehalase activity than 35S::TRE1 plants, they exhibited reduced growth compared to 35S::TRE1 seedlings (Van Houtte et al., 2013). Apparently, it is advantageous when the exogenous trehalose does not enter the cytoplasm, because the intracellular C-metabolism is not impaired by trehalose and trehalose-derived signals such as T6P.

The generation of glucose from trehalose presumably complements the exo mutant. The mutant could benefit from the generation of glucose for metabolic purposes. Alternatively, enhanced trehalase activity may trigger extracellular signaling processes.

Recent data revealed that trehalose metabolism rather than the trehalose itself is essential for proper stomatal function and survival of drought stress (Van Houtte et al., 2013). This finding was somewhat unanticipated, because trehalose was widely seen as a compatible solute and stress protectant (Vogel et al., 2001; Van Houtte et al., 2013). However, enhanced trehalase activity per se is not beneficial under all conditions. TRE1 overexpressors exhibited reduced growth in non-stress conditions (Van Houtte et al., 2013). Restricted growth of the exo mutant on sucrose did not result from elevated trehalase activity because 

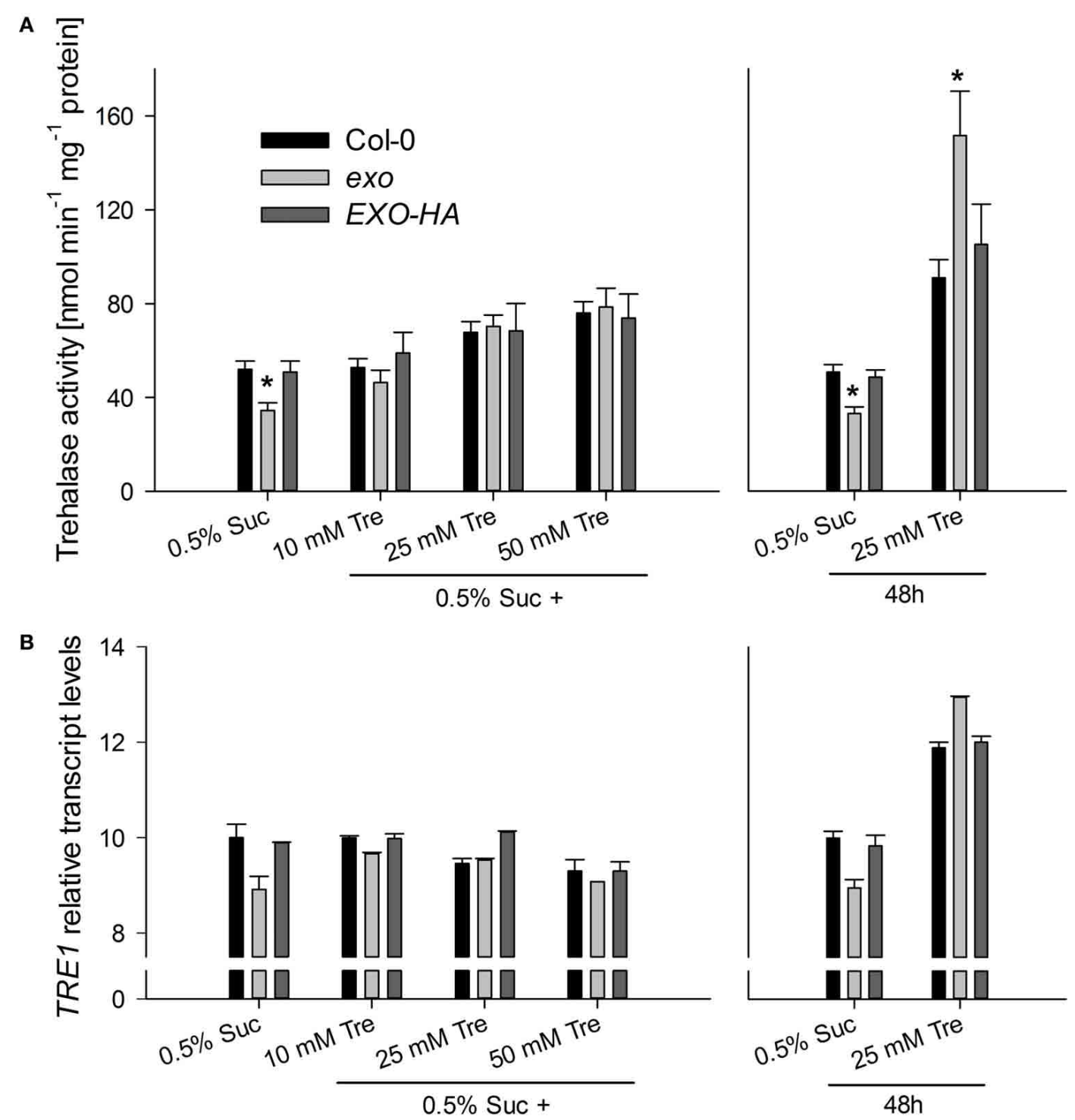

FIGURE 9 | Trehalase activity and TRE1 expression. (A) Wild-type, exo, and exo/35S::EXO-HA plants were established in half-concentrated MS medium supplemented with $0.5 \%$ sucrose for 5 days and transferred to medium supplemented with $0.5 \%$ sucrose plus $0,10,25$, or $50 \mathrm{mM}$ trehalose for 9 days. Alternatively, plants were established in half-concentrated MS medium supplemented with $0.5 \%$ sucrose. 12-day-old plants were transferred to medium supplemented with $0.5 \%$ sucrose with or without $25 \mathrm{mM}$ trehalose (Tre). Trehalase activity in shoot material is given as mean of three determinations \pm SE. Mutant values denoted with an asterisk are significantly different from those of their wild type ( $t$-test, $P<0.001$ ). Data are representative of three independent experiments with similar results. (B) Relative TRE1 transcript levels in shoots were given as described for Figure 2. Data are representative of three independent experiments with similar results. exo displayed reduced trehalase activity when grown on sucrose (Figure 9).

\section{CONCLUSIONS}

The phenotype of the exo mutants points to the importance of the extracellular C status. Although the apoplastic C-metabolism and $\mathrm{C}$-signaling processes are largely unknown, the altered sucrose and trehalose responses in exo suggest a role of the EXO protein in the control of the extracellular C-metabolism or C-signaling. Loss of EXO function causes reduced growth, impaired expression of starch-related and sugar-responsive genes, accumulation of ABA, anthocyanin, and starch. The phenotypic changes can partly be normalized by trehalose feeding. The poor understanding of extracellular processes complicate the understanding of EXO action. The availability of more data about these processes will allow building new hypothesis and moving toward targeted approaches.

\section{ACKNOWLEDGMENTS}

This work was supported by a grant of the Deutsche Forschungsgemeinschaft to Carsten Müssig (DFG, MU 1738/4-3). 


\section{REFERENCES}

Aghdasi, M., Schluepmann, H., and Smeekens, S. (2010). Characterization of Arabidopsis seedlings growth and development under trehalose feeding. J. Cell Mol. Res. 2, 1-9.

Alonso, J. M., Stepanova, A. N., Leisse, T. J., Kim, C. J., Chen, H., Shinn, P., et al. (2003). Genome-wide insertional mutagenesis of Arabidopsis thaliana. Science 301, 653-657. doi: 10.1126/science. 1086391

Arenas-Huertero, F., Arroyo, A., Zhou, L., Sheen, J., and León, P. (2000). Analysis of Arabidopsis glucose insensitive mutants, gin5 and gin6, reveals a central role of the plant hormone $\mathrm{ABA}$ in the regulation of plant vegetative development by sugar. Genes Dev. 14, 2085-2096. doi: 10.1101/gad.14.16. 2085

Baena-González, E., and Sheen, J. (2008). Convergent energy and stress signalling. Trends Plant Sci. 13, 474-482. doi: 10.1016/j.tplants.2008.06.006

Bailey-Serres, J., Fukao, T., Gibbs, D. J., Holdsworth, M. J., Lee, S. C., Licausi, F., et al. (2012). Making sense of low oxygen sensing. Trends Plant Sci. 17, 129-138. doi: 10.1016/j.tplants.2011.12.004

Boisson-Dernier, A., Kessler, S. A., and Grossniklaus, U. (2011). The walls have ears: the role of plant CrRLK1Ls in sensing and transducing extracellular signals. J. Exp. Bot. 62, 1581-1591. doi: 10.1093/jxb/erq445

Cheng, W.-H., Endo, A., Zhou, L., Penney, J., Chen, H.-C., Arroyo, A., et al. (2002). A unique shortchain dehydrogenase/reductase in Arabidopsis glucose signalling and abscisic acid biosynthesis and functions. Plant Cell 14, 2723-2743. doi: 10.1105/tpc.006494

Cheung, A. Y., and Wu, H.-M. (2011). THESEUS 1, FERONIA and relatives: a family of cell wallsensing receptor kinases. Curr. Opin. Plant Biol. 14, 632-641. doi: 10.1016/j.pbi.2011.09.001

Das, P. K., Shin, D. H., Choi, S.-B., and Park, Y.-I. (2012). Sugar-hormone cross-talk in anthocyanin biosynthesis. Mol. Cells 34, 501-507. doi: 10.1007/s10059-012-0151-x

Delatte, T. L., Sedijani, P., Kondou, Y., Matsui, M., de Jong, G. J., Somsen, G. W., et al. (2011). Growth arrest by trehalose-6-phosphate: an astonishing case of primary metabolite control over growth by way of the SnRK1 signaling pathway. Plant Physiol. 157, 160-174. doi: 10.1104/pp.111.180422
Eveland, A. L., and Jackson, D. P. (2012). Sugars, signaling, and plant development. J. Exp. Bot. 63, 3367-3377. doi: 10.1093/jxb/err379

Farré, E. M., and Weise, S. E. (2012). The interaction between the circadian clock and primary metabolism. Curr. Opin. Plant Biol. 15, 293-300. doi: 10.1016/j.pbi.2012.01.013

Frison, M., Parrou, J. L., Guillaumot, D., Masquelier, D., Francois, J., Chaumont, F., et al. (2007). The Arabidopsis thaliana trehalase is a plasma membrane-bound enzyme with extracellular activity. FEBS Lett. 581, 4010-4016. doi: 10.1016/j.febslet.2007.07.036

Gibon, Y., Pyl, E.-T., Sulpice, R., Lunn, J. E., Höhne, M., Günther, M., et al. (2009). Adjustment of growth, starch turnover, protein content and central metabolism to a decrease of the carbon supply when Arabidopsis is grown in very short photoperiods. Plant Cell Environ. 32, 859-874. doi: 10.1111/j.13653040.2009.01965.x

Gibson, S. I. (2000). Plant sugarresponse pathways. Part of a complex regulatory web. Plant Physiol. 124, 1532-1539. doi: 10.1104/pp.124.4.1532

Graf, A., Schlereth, A., Stitt, M., and Smith, A. M. (2010). Circadian control of carbohydrate availability for growth in Arabidopsis plants at night. Proc. Natl. Acad. Sci. U.S.A. 107, 9458-9463. doi: 10.1073/pnas.0914299107

Huijser, C., Kortstee, A., Pego, J., Weisbeek, P., Wisman, E., and Smeekens, S. (2000). The Arabidopsis SUCROSE UNCOUPLED-6 gene is identical to ABSCISIC ACID INSENSITIVE-4: involvement of abscisic acid in sugar responses. Plant J. 23, 577-585. doi: 10.1046/j.1365-313x.2000.00822.x

Laby, R. J., Kincaid, S., Kim, D., and Gibson, S. I. (2000). The Arabidopsis sugar-insensitive mutants sis4 and sis5 are defective in abscisic acid synthesis and response. Plant J. 23, 587-596. doi: 10.1046/j.1365-313x.2000.00833.x

Laxmi, A., Paul, L. K., Peters, J. L., and Khurana, J. P. (2004). Arabidopsis constitutive photomorphogenic mutant, blsl, displays altered brassinosteroid response and sugar sensitivity. Plant Mol. Biol. 56, 185-201. doi: 10.1007/s11103-004-2799-x

Leakey, A. D. B., Ainsworth, E. A., Bernacchi, C. J., Rogers, A., Long, S. P., and Ort, D. R. (2009). Elevated $\mathrm{CO}_{2}$ effects on plant carbon, nitrogen, and water relations: six important lessons from FACE.
J. Exp. Bot. 60, 2859-2876. doi: 10.1093/jxb/erp096

Levin, D. E. (2011). Regulation of cell wall biogenensis in Saccharomyces cerevisiae: the cell wall integrity signaling pathway. Genetics 189, 1145-1175. doi: 10.1534/genetics.111.128264

Lisso, J., Schröder, F., Fisahn, J., and Müssig, C. (2011). NFX1-LIKE2 (NFXL2) suppresses abscisic acid accumulation and stomatal closure in Arabidopsis thaliana. PLoS ONE 6:e26982. doi: 10.1371/journal.pone.0026982

Loreti, E., de Bellis, L., Alpi, A., and Perata, P. (2001). Why and how do plant cells sense sugars. Ann. Bot. 88, 803-812. doi: 10.1006/anbo.2001.1526

Lunn, J. E., Feil, R., Hendriks, J. H. M., Gibon, Y., Morcuende, R., Osuna, D., et al. (2006). Sugar-induced increases in trehalose 6-phosphate are correlated with redox activation of ADPglucose pyrophosphorylase and higher rates of starch synthesis in Arabidopsis thaliana. Biochem. J. 397, 139-148. doi: 10.1042/BJ20060083

Müller, J., Aeschbacher, R. A., Wingler, A., Boller, T., and Wiemken, A. (2001). Trehalose and trehalase in Arabidopsis. Plant Physiol. 125, 1086-1093. doi: 10.1104/pp.125.2.1086

Nakagawa, T., Kurose, T., Hino, T., Tanaka, K., Kawamukai, M., Niwa, Y., et al. (2007). Development of series of Gateway binary vectors, pGWBs, for realizing efficient construction of fusion genes for plant transformation. J. Biosci. Bioeng. 104, 34-41. doi: 10.1263/jbb.104.34

Németh, K., Salchert, K., Putnoky P., Bhalerao, R., Koncz-Kálmán, Z., Stankovic-Stangeland, B., et al. (1998). Pleiotropic control of glucose and hormone responses by PRL1, a nuclear WD protein, in Arabidopsis Genes Dev. 12, 3059-3073. doi: 10.1101/gad.12.19.3059

Pego, J. V., Weisbeek, P. J., and Smeekens, S. C. M. (1999). Mannose inhibits Arabidopsis germination via a hexokinasemediated step. Plant Physiol. 119, 1017-1024. doi: 10.1104/pp.119.3. 1017

Robaglia, C., Thomas, M., and Meyer, C. (2012). Sensing nutrient and energy status by SnRK1 and TOR kinases. Curr. Opin. Plant Biol. 15, 301-307. doi: 10.1016/j.pbi.2012.01.012

Rolland, F., Baena-González, E., and Sheen, J. (2006). Sugar sensing and signalling in plants: conserved and novel mechanisms. Annu. Rev. Plant Biol. 57, 675-709. doi: 10.1146/ annurev.arplant.57.032905.105441

Ruts, T., Matsubara, S., WieseKlinkenberg, A., and Walter, A. (2012). Aberrant temporal growth pattern and morphology of root and shoot caused by a defective circadian clock in Arabidopsis thaliana. Plant J. 72, 154-161. doi: 10.1111/j.1365313X.2012.05073.X

Schluepmann, H., Berke, L., and Sanchez-Perez, G. F. (2012). Metabolism control over growth: a case for trehalose-6-phosphate in plants. J. Exp. Bot. 63, 3379-3390. doi: $10.1093 / \mathrm{jxb} / \mathrm{err} 311$

Schluepmann, H., van Dijken, A., Aghdasi, M., Wobbes, B., Paul., M., and Smeekens, S. (2004). Trehalose mediated growth inhibition of Arabidopsis seedlings is due to trehalose-6-phosphate accumulation. Plant Physiol. 135, 879-890. doi: 10.1104/pp.104.039503

Schröder, F., Lisso, J., Lange, P., and Müssig, C. (2009). The extracellular EXO protein mediates cell expansion in Arabidopsis leaves. BMC Plant Biol. 9:20. doi: 10.1186/14712229-9-20

Schröder, F., Lisso, J., and Müssig, C. (2011). EXORDIUM-LIKE1 promotes growth during low carbon availability in Arabidopsis. Plant Physiol. 156, 1620-1630. doi: 10.1104/pp.111.177204

Schröder, F., Lisso, J., and Müssig, C. (2012). Expression pattern and putative function of EXL1 and homologous genes in Arabidopsis. Plant Signal. Behav. 7, 22-27. doi: 10.4161/psb.7.1.18369

Sebastian, S., and Zeeman, S. C. (2012). Starch Metabolism in Arabidopsis. The Arabidopsis Book 9:e0160. doi: 10.1199/tab.0160

Smeekens, S., Ma, J., Hanson, J., and Rolland, F. (2010). Sugar signals and molecular networks controlling plant growth. Curr. Opin. Plant Biol. 13, 274-279. doi: 10.1016/j.pbi.2009.12.002

Stitt, M., and Zeeman, S. C. (2012). Starch turnover: pathways, regulation and role in growth. Curr. Opin. Plant Biol. 15, 282-292. doi: 10.1016/j.pbi.2012.03.016

Teng, S., Keurentjes, J., Bentsink, L., Koornneef, M., and Smeekens, S. (2005). Sucrose-specific induction of anthocyanin biosynthesis in Arabidopsis requires the MYB75/PAP1 gene. Plant Physiol. 139, 1840-1852. doi: 10.1104/pp.105.066688

Van Houtte, H., Vandesteene, L., López-Galvis, L., Lemmens, L., 
Kissel, E., Carpentier, S., et al. (2013). Overexpression of the trehalase gene AtTRE1 leads to increased drought stress tolerance in Arabidopsis and is involved in abscisic acid-induced stomatal closure. Plant Physiol. 161, 1158-1171. doi: 10.1104/pp.112.211391

Veyres, N., Danon, A., Aono, M., Galliot, S., Karibasappa, Y. B., Diet, A., et al. (2008). The Arabidopsis sweetie mutant is affected in carbohydrate metabolism and defective in the control of growth, development and senescence. Plant J. 55, 665-686. doi: 10.1111/j.1365313X.2008.03541.x

Vogel, G., Fiehn, O., Jean-Richard-ditBressel, L., Boller, T., Wiemken, A., Aeschbacher, R. A., et al. (2001). Trehalose metabolism in
Arabidopsis: occurrence of trehalose and molecular cloning and characterization of trehalose-6phosphate synthase homologues. J. Exp. Bot. 52, 1817-1826. doi: 10.1093/jexbot/52.362.1817

Wingler, A., Fritzius, T., Wiemken, A., Boller, T., and Aeschbacher, R. A. (2000). Trehalose induces the ADP-glucose pyrophosphorylase gene, $A p L 3$, and starch synthesis in Arabidopsis. Plant Physiol. 124, 105-114. doi: 10.1104/pp.124.1.105

Wolf, S., Hématy, K., and Höfte, H. (2012). Growth control and cell wall signalling in plants. Annu. Rev. Plant Biol. 63, 381-407. doi: 10.1146/annurev-arplant-042811105449

Zhang, Y., Primavesi, L. F., Jhurreea, D., Andralojc, P. J., Mitchell, R. A. C.,
Powers, S. J., et al. (2009). Inhibition of SNF1-related protein kinasel activity and regulation of metabolic pathways by trehalose-6-phosphate. Plant Physiol 149, 1860-1871. doi: 10.1104/pp.108.133934

Zhou, L., Jang, J.-C., Jones, T. L., and Sheen, J. (1998). Glucose and ethylene signal transduction crosstalk revealed by an Arabidopsis glucoseinsensitive mutant. Proc. Natl. Acad. Sci. U.S.A. 95, 10294-10299. doi: $10.1073 /$ pnas.95.17.10294

Conflict of Interest Statement: The authors declare that the research was conducted in the absence of any commercial or financial relationships that could be construed as a potential conflict of interest.
Received: 10 April 2013; accepted: 06 June 2013; published online: 25 June 2013.

Citation: Lisso J, Schröder F and Müssig $C$ (2013) EXO modifies sucrose and trehalose responses and connects the extracellular carbon status to growth. Front. Plant Sci. 4:219. doi: 10.3389/fpls. 2013.00219

This article was submitted to Frontiers in Functional Plant Ecology, a specialty of Frontiers in Plant Science.

Copyright (c) 2013 Lisso, Schröder and Müssig. This is an open-access article distributed under the terms of the Creative Commons Attribution License, which permits use, distribution and reproduction in other forums, provided the original authors and source are credited and subject to any copyright notices concerning any third-party graphics etc. 


\section{APPENDIX}

Table A1 | Primer sequences used for quantitative RT-PCR.

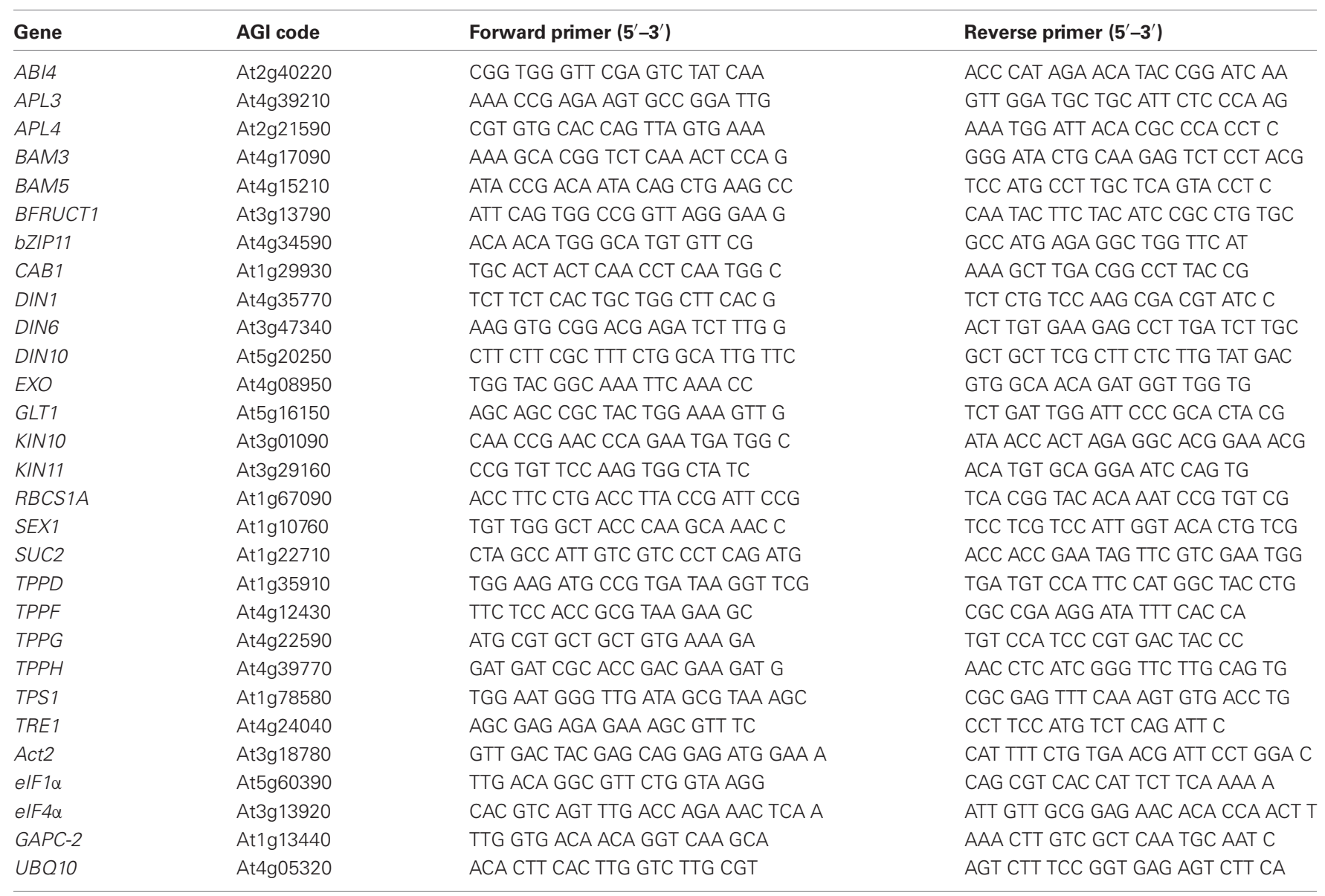

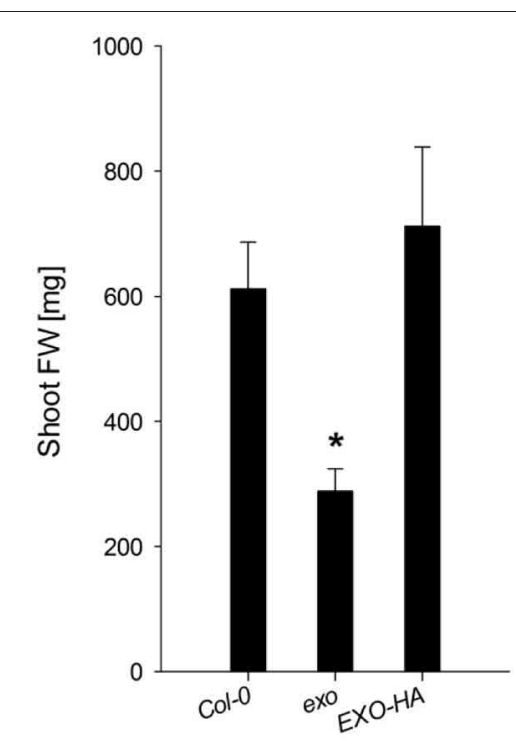

FIGURE A1 | Shoot fresh weight of wild-type, exo, and exo/35S::EXO-HA plants. Plants were grown in soil under long-day conditions and harvested after 35 days. The mutant value denoted with an asterisk is significantly different from the wild-type value ( $t$-test, $P<0.001)$. 


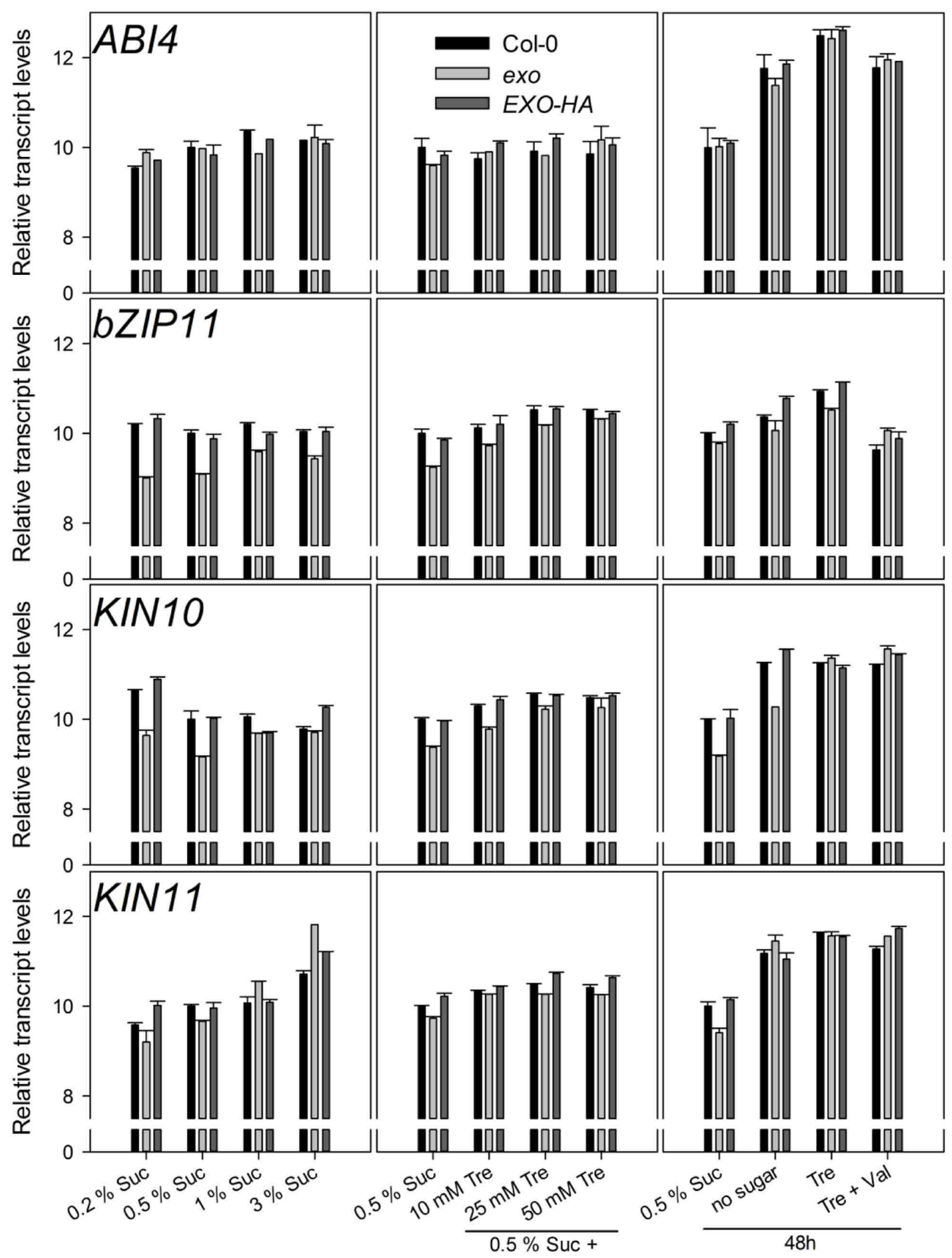

FIGURE A2 | Relative transcript levels of the ABI4, bZIP11, KIN10, and $\boldsymbol{K I N 1 1}$ genes. Experimental details are described in Figure 2. 

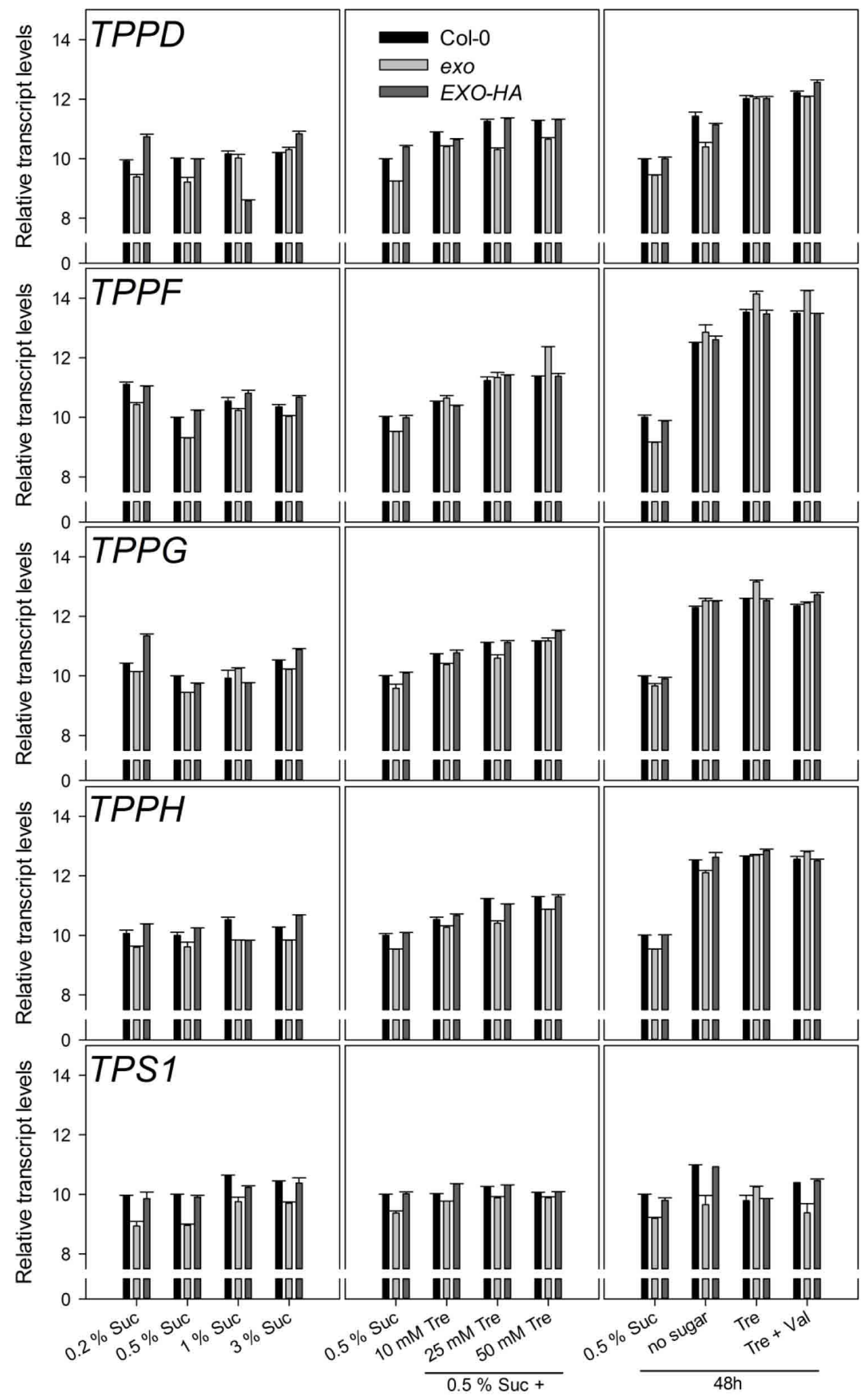

FIGURE A3 | Relative transcript levels of trehalose-related genes. Experimental details are described in Figure 2. 

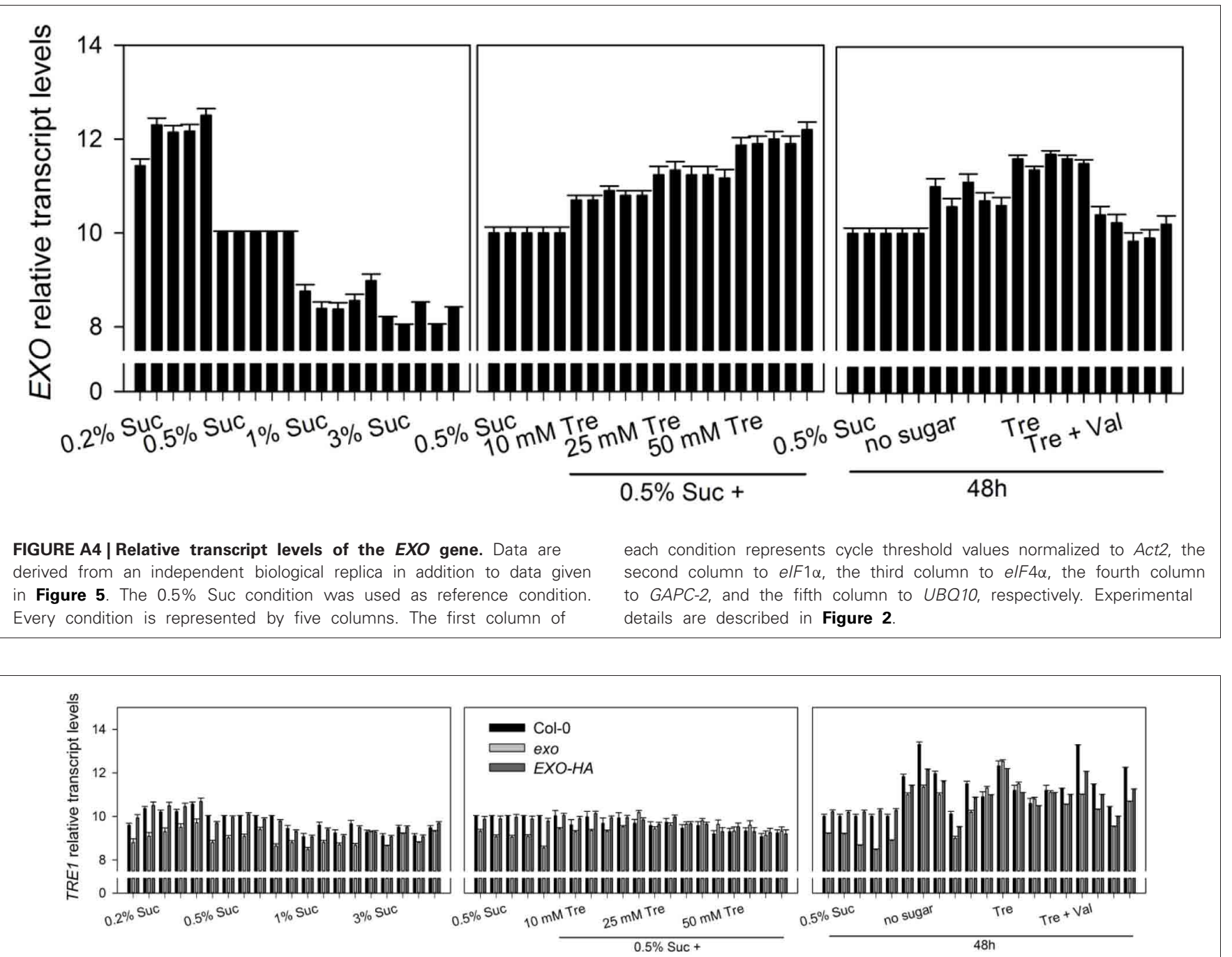

FIGURE A5 | Relative transcript levels of the TRE1 gene. Data are derived from an independent biological replica in addition to data given in Figure $\mathbf{9}$. Each condition is represented by five sets of three columns (Col-0, exo, and EXO-HA). The first set of columns per condition represents cycle threshold values normalized to $A c t 2$, the second set of columns to elF1 $\alpha$, the third set of columns to elF4 $\alpha$, the fourth set of columns to GAPC-2, and the fifth set of columns to $U B Q 10$, respectively. Experimental details are described in Figure 2. 